\title{
The Changing Epidemiology of Gastroesophageal Reflux Disease: Are Patients Getting Younger?
}

\author{
Takahisa Yamasaki, ${ }^{1}$ Colin Hemond ${ }^{2}$ Mohamed Eisa, ${ }^{3}$ Stephen Ganocy, ${ }^{4}$ and Ronnie Fass ${ }^{1 *}$ \\ ${ }^{I}$ The Esophageal and Swallowing Center, Division of Gastroenterology and Hepatology, MetroHealth Medical Center, Case Western Reserve \\ University, Cleveland, OH, USA; ${ }^{2}$ Department of Physiology and Biophysics, Case Western Reserve University, Cleveland, OH, USA; ${ }^{3}$ Department \\ of Internal Medicine, Case Western Reserve University, MetroHealth Medical Center, Cleveland, OH, USA; and ${ }^{4}$ Center for Health Care Research \\ and Policy, Case Western Reserve University, MetroHealth Medical Center, Cleveland, OH, USA
}

\section{Background/Aims}

Gastroesophageal reflux disease (GERD) is a common disease globally with increasing prevalence and consequently greater burden on the Healthcare system. Traditionally, GERD has been considered a disease of middle-aged and older people. Since risk factors for GERD affect a growing number of the adult population, concerns have been raised that increasingly younger people may develop GERD. We aim to determine if the proportion of younger patients has increased among the GERD population.

\section{Methods}

The incidence of GERD as well as several variables were evaluated during an 11-year period. Explorys was used to evaluate datasets at a "Universal" and Healthcare system in northern Ohio to determine if trends at a local level reflected those at a universal level. GERD patients were classified into 7 age groups (15-19, 20-29, 30-39, 40-49, 50-59, 60-69, and $\geq 70$ years).

\section{Results}

The proportion of patients with GERD increased in all age groups, except for those who were $\geq 70$ years in the universal dataset $(P$ $<0.001)$ and those who were $\geq 60$ years in the Healthcare system $(P<0.001)$. The greatest rise was seen in 30-39 years in both datasets $(P<0.001)$. Similarly, the proportion of GERD patients who were using proton pump inhibitors increased in all age groups except for those who were $\geq 70$ years in both datasets $(P<0.001)$, with the greatest increase being the group 30-39 years $(P<$ $0.001)$.

\section{Conclusion}

Over the last decade, there has been a significant increase in the proportion of younger patients with GERD, especially those within the age range of 30-39 years.

(J Neurogastroenterol Motil 2018;24:559-569)

\section{Key Words}

Age; Epidemiology; Gastroesophageal reflux disease; Proton pump inhibitors

Received: August 7, 2018 Revised: August 22, 2018 Accepted: August 28, 2018

(a) This is an Open Access article distributed under the terms of the Creative Commons Attribution Non-Commercial License (http://creativecommons. org/licenses/by-nc/4.0) which permits unrestricted non-commercial use, distribution, and reproduction in any medium, provided the original work is properly cited.

*Correspondence: Ronnie Fass, MD, FACG

Division of Gastroenterology and Hepatology, Esophageal and Swallowing Center, Case Western Reserve University, MetroHealth Medical Center, 2500 MetroHealth Drive, Cleveland, OH 44109, USA

Tel: +1-216-778-3145, Fax: +1-216-957-8410, E-mail: ronnie.fass@gmail.com 


\section{Introduction}

Gastroesophageal reflux disease (GERD) is a common disease that can cause troublesome symptoms and have a significant impact on quality of life. ${ }^{1}$ GERD is a chronic and highly prevalent disorder. A recent systematic review showed that the prevalence of GERD is $18.1-27.8 \%$ in North America, 8.8-25.9\% in Europe, 2.5-7.8\% in East Asia, 8.7-33.1\% in the Middle East, $11.6 \%$ in Australia, and $23.0 \%$ in South America. ${ }^{2}$ The increase in GERD prevalence may be due to multiple factors such as older age, male sex, race, intake of analgesics, consumption of certain types of food and drinks, decrease in the prevalence of Helicobacter pylori infection, smoking, family history of GERD, high body mass index (BMI), and limited physical activity. These risk factors are mostly related to a patient's lifestyle. ${ }^{3-5}$

Aging has been consistently associated with an increased risk for GERD symptoms. Some studies have reported that the effects of aging on esophageal and esophagogastric junction mechanophysical properties of patients with GERD may explain the age effect. ${ }^{6-8}$ Esophageal peristalsis may decrease, and esophageal acid exposure and anatomical disruption of the esophagogastric junction may increase with aging. ${ }^{9}$ In a meta-analysis, the prevalence of GERD symptoms was higher in subjects aged $\geq 50$ years $(\mathrm{OR}, 1.32 ; 95 \%$, CI, 1.12-1.54) but with significant heterogeneity between studies $\left(\mathrm{I}^{2}\right.$ $=91.5 \%, P<0.001)$, indicating substantial heterogeneity among study results. ${ }^{10}$ In addition, aging was associated with increased hospitalizations for erosive esophagitis and GERD complications. ${ }^{11}$ However, in the late 1980s, aging was inversely correlated with hospitalization for esophagitis (OR for $>85$ years [65-69 years: the reference], 0.66; 95\% CI, 0.65-0.67). ${ }^{12}$ This reversal in association of age with erosive esophagitis suggests a cohort effect.

Johnson and Fennerty ${ }^{13}$ analyzed 11945 patients with erosive esophagitis who were enrolled in 5 prospective, randomized, controlled clinical trials. The authors assessed the relationship between age, severe heartburn symptoms, and severe erosive esophagitis. The study demonstrated that severe erosive esophagitis became more prevalent with advancing age. Only $12 \%$ of patients aged $<$ 21 years showed severe erosive esophagitis as compared with $37 \%$ in those aged $>70$ years. The OR of having severe esophagitis increased by 1.17 (95\% CI, 1.13-1.20) for each decade of age $(P<$ $0.001)$.

As risk factors for GERD increasingly affect the general population, concerns have been raised that more young individuals will develop GERD and its potential consequences. Thus, the aim of this study is to determine if GERD is becoming more prevalent in younger populations than in older populations by assessing the proportion of patients with GERD in each age group using a population-based data and comparing the results with a major Healthcare system.

\section{Materials and Methods}

\section{Patients}

A population-based analysis of the Explorys dataset (Explorys Inc, Cleveland, OH, USA; http://www.explorys.com) was performed. Explorys is an aggregate electronic medical record database representing over 54 million patients from 26 institutions throughout the United States of America. De-identified data were obtained using the explore application of the Explorys platform. This placed a Healthcare gateway server behind the firewall of each participating Healthcare institution. The de-identified data were collected through billing inquiries that included diagnosis, findings, procedures, electronic health records, laboratory systems, etc. This was then processed and passed on to a data grid. A web application allowed each Healthcare organization to search and analyze the aggregated, standardized, normalized, and de-identified population level data. All data were de-identified to meet the Health Insurance Portability and Accountability Act (HIPPA) and Health Information Technology for Economic and Clinical Health (HITECH) Act standards. Therefore, this study was deemed not to be human subject research by the Institutional Review Board of the MetroHealth System. Business affiliation agreements are in place between all participating Healthcare systems and Explorys Inc. regarding contribution of electronic health records data and the use of deidentified data. Unified Medical Language Systems (UMLS) ontologies were used to map electronic health records data to facilitate searching and indexing. Diagnosis, findings, and procedures were mapped into the systematized nomenclature of medicalclinical terms (SNOMED-CT) hierarchy. Prescription medication orders were mapped to RxNorm. Laboratory test observations were mapped to logistical observation identifier names and codes (LOINC) established by the Regenstrief Institute. Through the Explorys cohort definition feature, subjects were identified by adding "Diagnosis: Gastroesophageal reflux disease" as the cohort criterion, which was determined by patients' providers. GERD patients were stratified into 7 age groups by years (15-19, 20-29, $30-39,40-49,50-59,60-69$, and $\geq 70$ ). Patients younger than aged 15 years were excluded from the analysis. Secular trends of GERD 
proportion were assessed for each age group.

\section{Study Design}

This was a large nationwide database cohort study using the Explorys dataset. Case identification was carried out using an electronic health search. The diagnosis information was obtained from multiple sources, making it a sensitive tool. The term "gastroesophageal reflux disease" was used in the Explorys search tool. Proportion of GERD as well as several variables such as age, sex, race, $\mathrm{BMI}$, and treatment with a proton pump inhibitor (PPI) were evaluated during an 11-year period from 2006 to 2016. Using Explorys, we examined the universal patient population in the universal dataset and compared it with the patient population of a large Healthcare system in Ohio. Patient demographics, symptoms, associated conditions, and treatments were identified using a search tool. We also stratified patients into the following 5 groups with regard to BMI: underweight (BMI $\left.<18.5 \mathrm{~kg} / \mathrm{m}^{2}\right)$, normal $(18.5$ $\left.\mathrm{kg} / \mathrm{m}^{2} \leq \mathrm{BMI}<25 \mathrm{~kg} / \mathrm{m}^{2}\right)$, overweight $\left(25 \mathrm{~kg} / \mathrm{m}^{2} \leq \mathrm{BMI}<30\right.$ $\mathrm{kg} / \mathrm{m}^{2}$ ), obese (BMI $30 \mathrm{~kg} / \mathrm{m}^{2} \leq$ BMI $<40 \mathrm{~kg} / \mathrm{m}^{2}$ ), and severely obese $\left(\mathrm{BMI} \geq 40 \mathrm{~kg} / \mathrm{m}^{2}\right)$. Cell counts in Explorys were rounded to the nearest 10 .

\section{Statistical Methods}

Most demographic data in this study that were extracted from Explorys were categorical and were thus presented as counts and percentages. Differences across age groups for proportion of GERD and proportion of PPI usage in 2006 compared with 2016 were tested using a 2-way contingency table chi-square test. The Cochran-Armitage test was used to determine if there was a yearby-year trend in the proportion of GERD and PPI usage for the 11-year period from 2006 through 2016. Statistical significance was established at $P<0.05$ throughout the study. SAS version 9.4 (World Headquarters SAS Institute Inc, Cary, NC, USA) was used to analyze all statistical tests.

\section{Results}

\section{Universal Explorys Dataset}

Table 1 shows the demographics of GERD patients using the universal Explorys dataset, that the total number of patients diagnosed with GERD in a given year between 2006 and 2016 had increased from 2006 (179 300 patients) to 2014 (1 113910 patients) but decreased in 2015 (1 113160 patients) and 2016 (1 032140 patients). The increase in number of GERD patients represents a growing number of health centers using the Epic electronic medical record and their datasets were incorporated into Explorys universal.

Analysis of GERD diagnosis by age groups revealed that from 2006 to 2016 the proportion of GERD patients over the age 70 $(\geq 70)$ and diagnosed with GERD had significantly decreased $(-10.6 \%, P<0.001)$ (Fig. 1A), while the younger age groups demonstrated a significant increase in the proportion of GERD patients over the same period of time $(15-19$ : $0.2 \%, P<0.001$; 20-29: 2.4\%, $P<0.001$; 30-39: 3.2\%, $P<0.001$; 40-49: 2.8\%, $P<0.001$; 50-59: 2.5\%, $P<0.001$; 60-69: 0.8\%, $P<0.001)$. The greatest increase in the proportion of patients with GERD was noted in the 30-39 years age group (Fig. 2A).

The proportion of patients with GERD by sex has been consistent from 2006 to 2016: 60.0\% of the patients with GERD were women, and $40.0 \%$ were men. While the majority of the GERD patients were Caucasian, the proportion of Caucasian patients with GERD had increased from $76.1 \%$ in 2006 to a peak of $80.7 \%$ in 2015 , followed by a slight decrease to $80.4 \%$ in 2016 . African Americans account for the second-highest ethnicity among GERD patients. However, the proportion of GERD patients in this group has decreased over time from $16.5 \%$ in 2006 to $11.9 \%$ in 2016. The majority of patients with GERD were considered obese (45.4\%) or severely obese $(30.8 \%)$.

When examining PPI usage in patients with GERD by age group, results were similar to distribution of GERD diagnosis (Fig. $3 \mathrm{~A})$. The proportion of GERD patients using PPIs has declined significantly in the age group $\geq 70$ years between 2006 and 2016 $(-11.4 \%, P<0.001)$, while the proportion of GERD patients using PPIs in the other remaining age groups has increased significantly (15-19 years: $0.2 \%, P<0.001 ; 20-29$ years: $2.0 \%, P$ $<0.001$; 30-39 years: $3.5 \%, P<0.001$; $40-49$ years: $3.5 \%, P$ $<0.001 ; 50-59$ years: $2.8 \%, P<0.001 ; 60-69$ years: $0.3 \%, P$ $<0.001)$. The highest increase was noted in the 30-39 years age group (Fig. 4A).

\section{Healthcare System}

The data collected from patients affiliated with the Northern Ohio Healthcare system showed results similar to the universal dataset. Table 2 demonstrates the demographics of GERD patients at the Healthcare system. The total number of GERD diagnoses in each year had increased $108.0 \%$ from 2006 (9110 patients) to 2015 (18 950 patients). In 2016, the total number of patients diagnosed with GERD decreased slightly to 18460 patients (2.6\%). As previously mentioned, the total numbers represent inclusion of new Epic users into the database. 


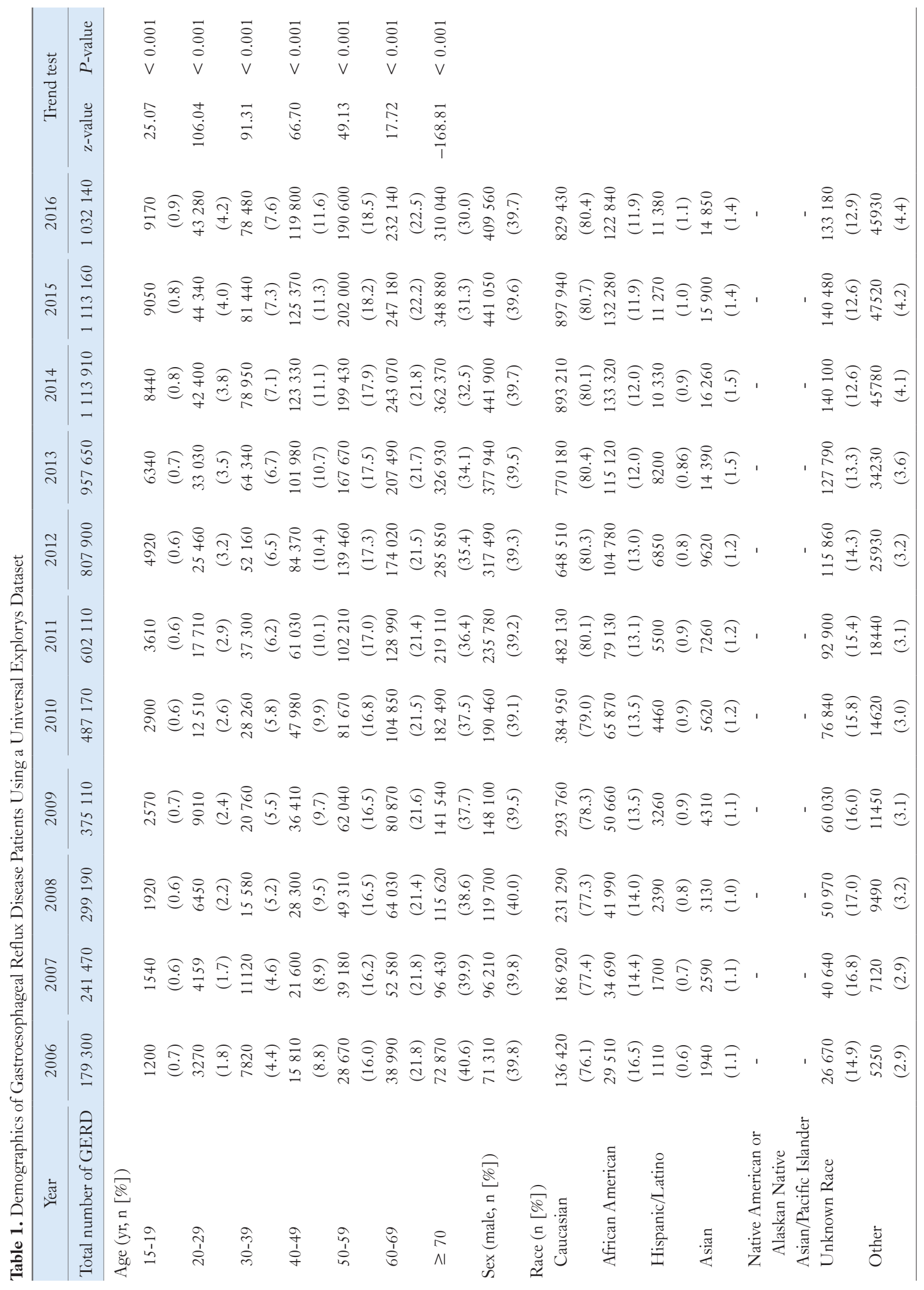




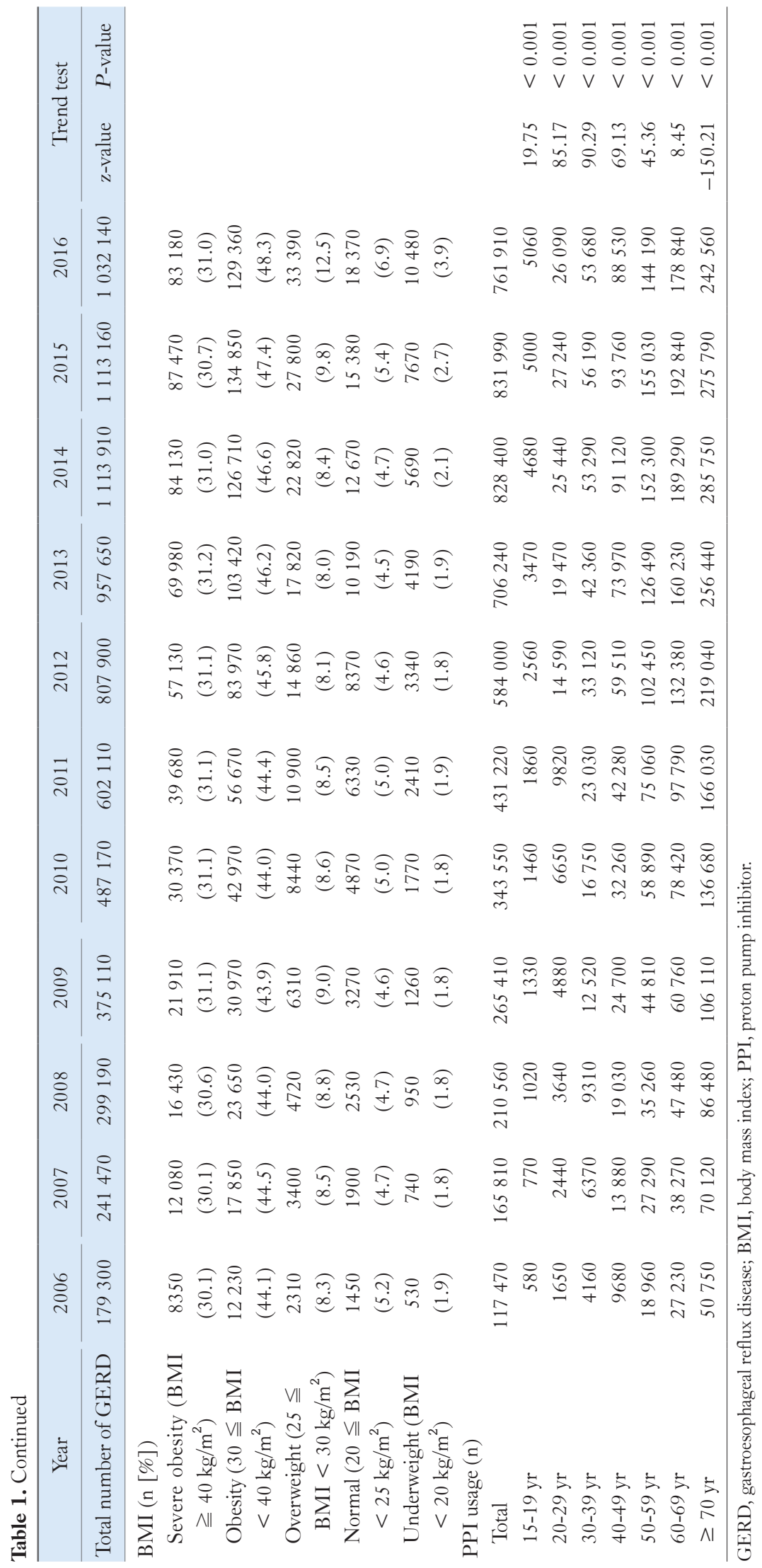



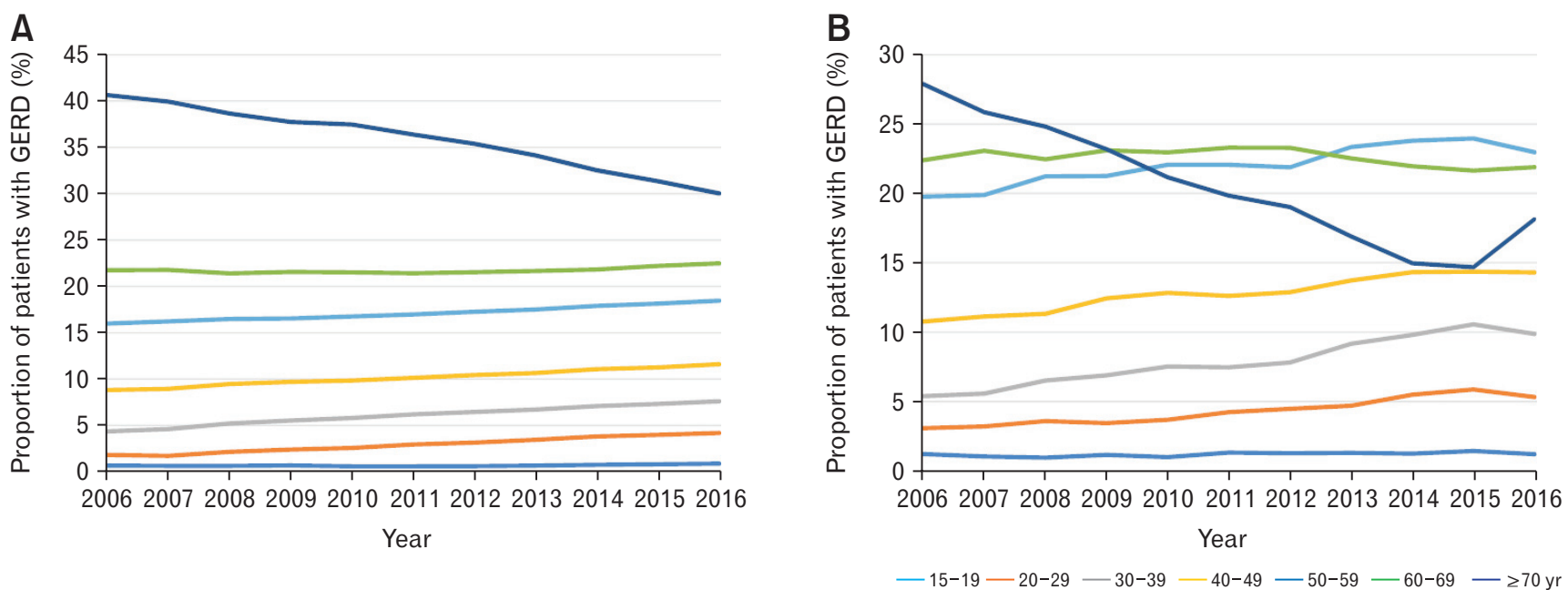

Figure 1. The proportion of patients with gastroesophageal reflux disease (GERD) by age group between 2006 and 2016 using universal Explorys dataset (A) and Healthcare system dataset (B).
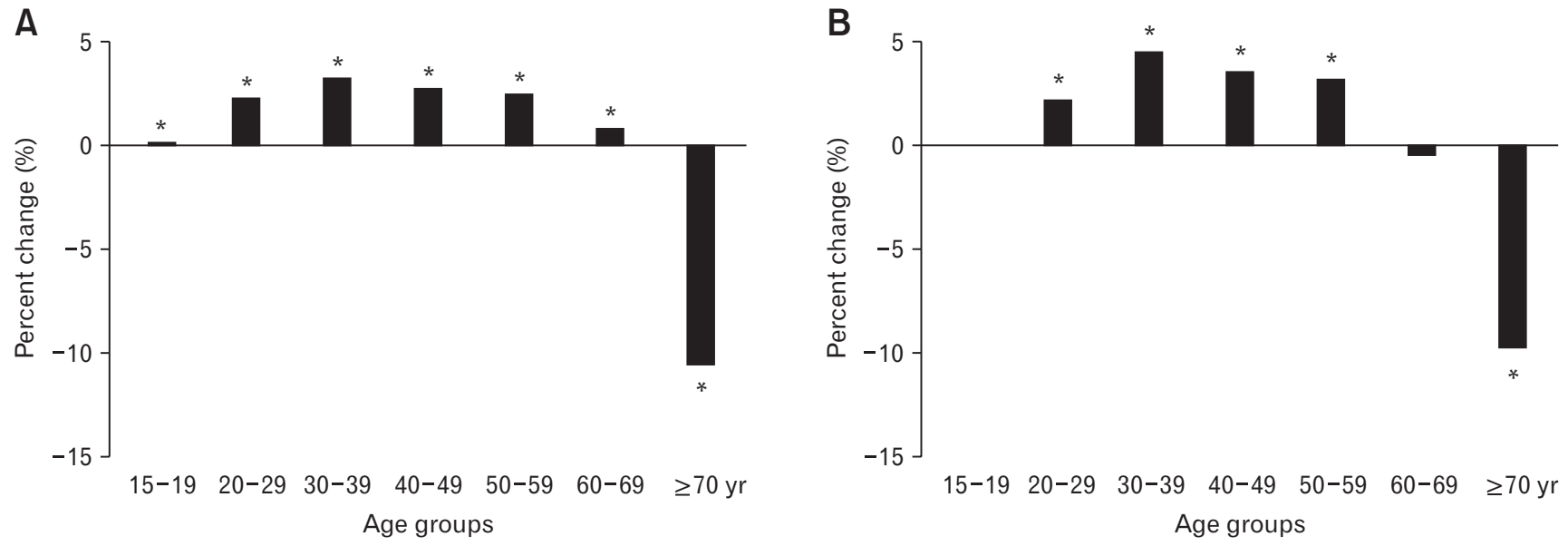

Figure 2. Percent of change in the proportion of patients with gastroesophageal reflux disease (GERD) between 2006 and 2016 using universal Explorys dataset (A) and Healthcare system dataset (B). ${ }^{*} P<0.001$.

The proportion of patients with GERD by sex has been fairly consistent. When viewing GERD diagnoses in a given year, the proportion ranged from $63.0 \%$ to $66.9 \%$ for women and from $33.0 \%$ to $37.1 \%$ for men. Most patients diagnosed with GERD are Caucasian, followed by African Americans, which is similar to that seen in the Universal dataset. The Healthcare system dataset had a population of Native Americans/Alaskan Natives (0.1-0.3\%) and Asian/Pacific Islanders (0.1-0.2\%). However, Native Americans/ Alaskan Natives or Asian/Pacific Islanders were not mentioned in the Universal dataset due to their very small population size relative to the other ethnicities who composed the GERD population. There was no clear trend in GERD incidence by race. While the Universal dataset showed that most patients diagnosed with GERD were obese (44.0-48.0\%), the Healthcare system data showed that most patients were severely obese (45.0-49.0\%).

The proportion of patients with GERD was also analyzed, and it was found that most patients were aged $\geq 70$ years (Fig. 1B). As with the Universal dataset, the proportion of patients diagnosed with GERD in a given year who are aged $\geq 70$ years has been decreasing from 2006 to 2016 . In addition, the proportion of patients with GERD diagnosis who were aged $\geq 70$ years dropped significantly by $9.7 \%(P<0.001)$. The proportion of patients with GERD in the 60-69 years age group demonstrated a slight decrease $(-0.5 \%, P=0.347)$. The proportion of patients with GERD in the remaining age groups showed a significant increase (15-19 years: $0.02 \%, P=0.957 ; 20-29$ years: $2.2 \%, P<0.001$; 


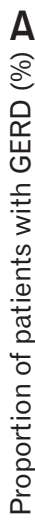

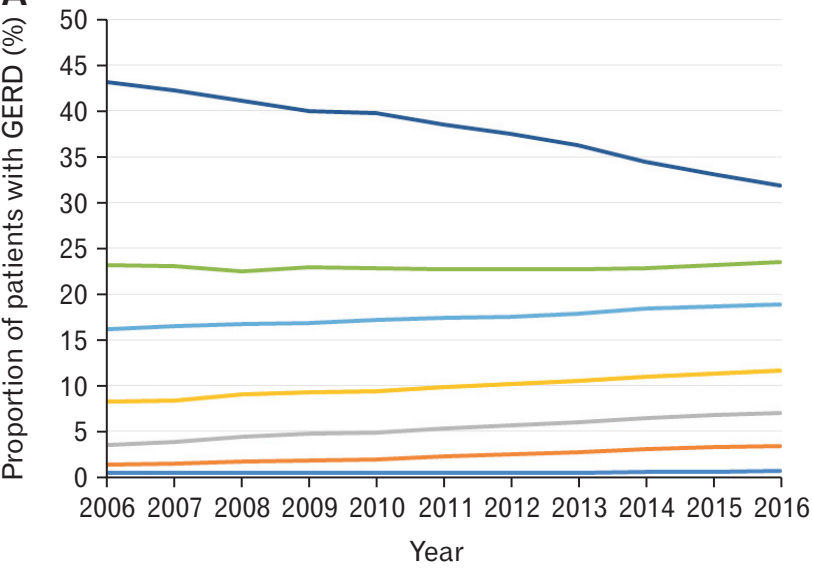

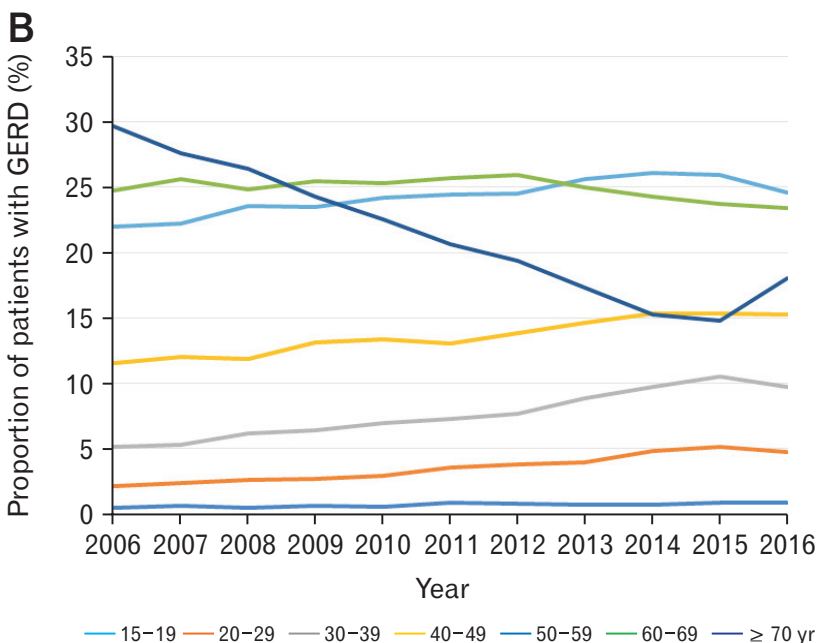

Figure 3. The proportion of gastroesophageal reflux disease (GERD) patients using proton pump inhibitors by age group between 2006 and 2016 using universal Explorys dataset (A) and Healthcare system dataset (B).
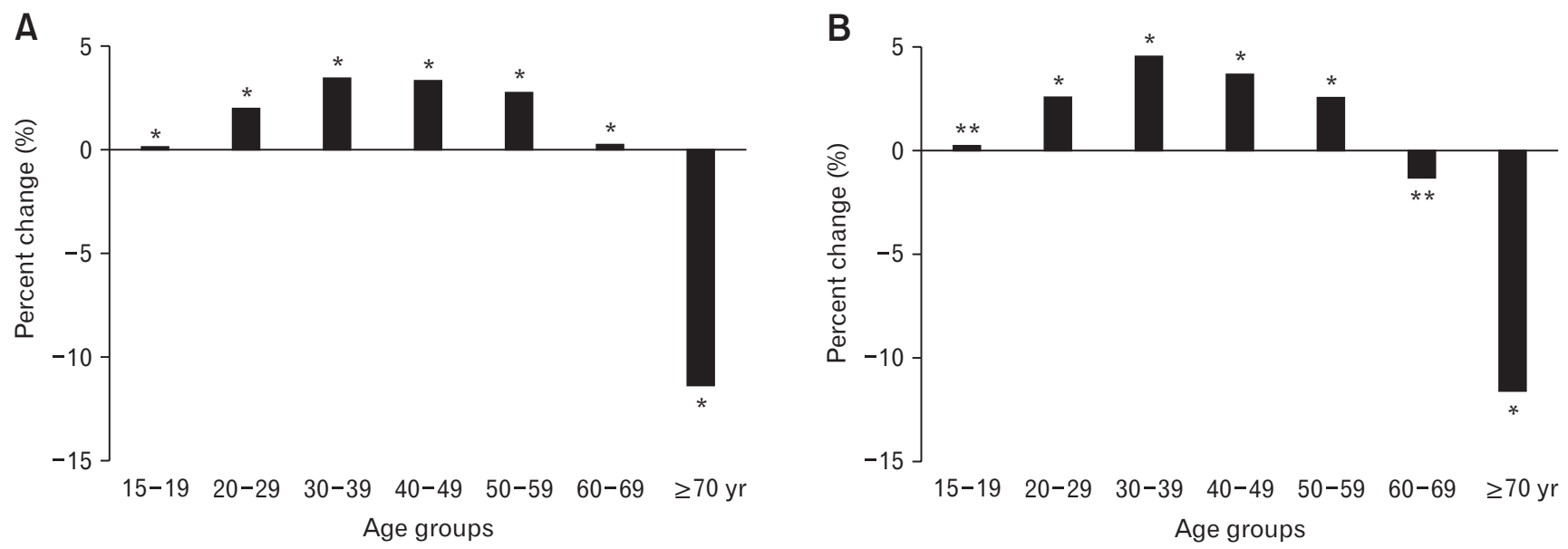

Figure 4. Percent of change in the proportion of gastroesophageal reflux disease patients using proton pump inhibitors between 2006 and 2016 using universal Explorys dataset (A) and Healthcare system dataset (B). ${ }^{*} P<0.001,{ }^{* *} P<0.05$.

30-39 years: $4.5 \%, P<0.001 ; 40-49$ years: $3.5 \%, P<0.001 ; 50$ 59 years: $2.6 \%, P<0.001$ ) (Fig. 2 B).

When examining PPI usage in GERD patients by age group, the results were also similar to the proportion of patients with GERD (Fig. 3B). The proportion of patients with GERD using PPIs has decreased significantly from 2006 to 2016 in the aged $\geq 70$ years $(-11.6 \%, P<0.001)$ and the aged $60-69$ years group $(-1.3 \%, P=0.029)$. The proportion of patients with GERD who were using PPIs, has increased significantly (aged 15-19: 0.3\%, $P=0.010$; aged 20-29: 2.7\%, $P<0.001$; aged 30-39: 4.6\%, $P$ $<0.001$; aged 40-49: 3.8\%, $P<0.001$; aged 50-59: $2.6 \%, P<$ 0.001) (Fig. 4B).

\section{Discussion}

The present study is a large, population-based cohort study that examined if GERD is becoming more common among younger populations when using trend analysis. In general, the number of patients with GERD in our study has increased over time. However, the observed numerical increase in patients in the dataset represents an increase in the number of registered institutions in Explorys.

Our study showed that the proportion of patients with GERD has increased in all age groups, with the exception of those who 


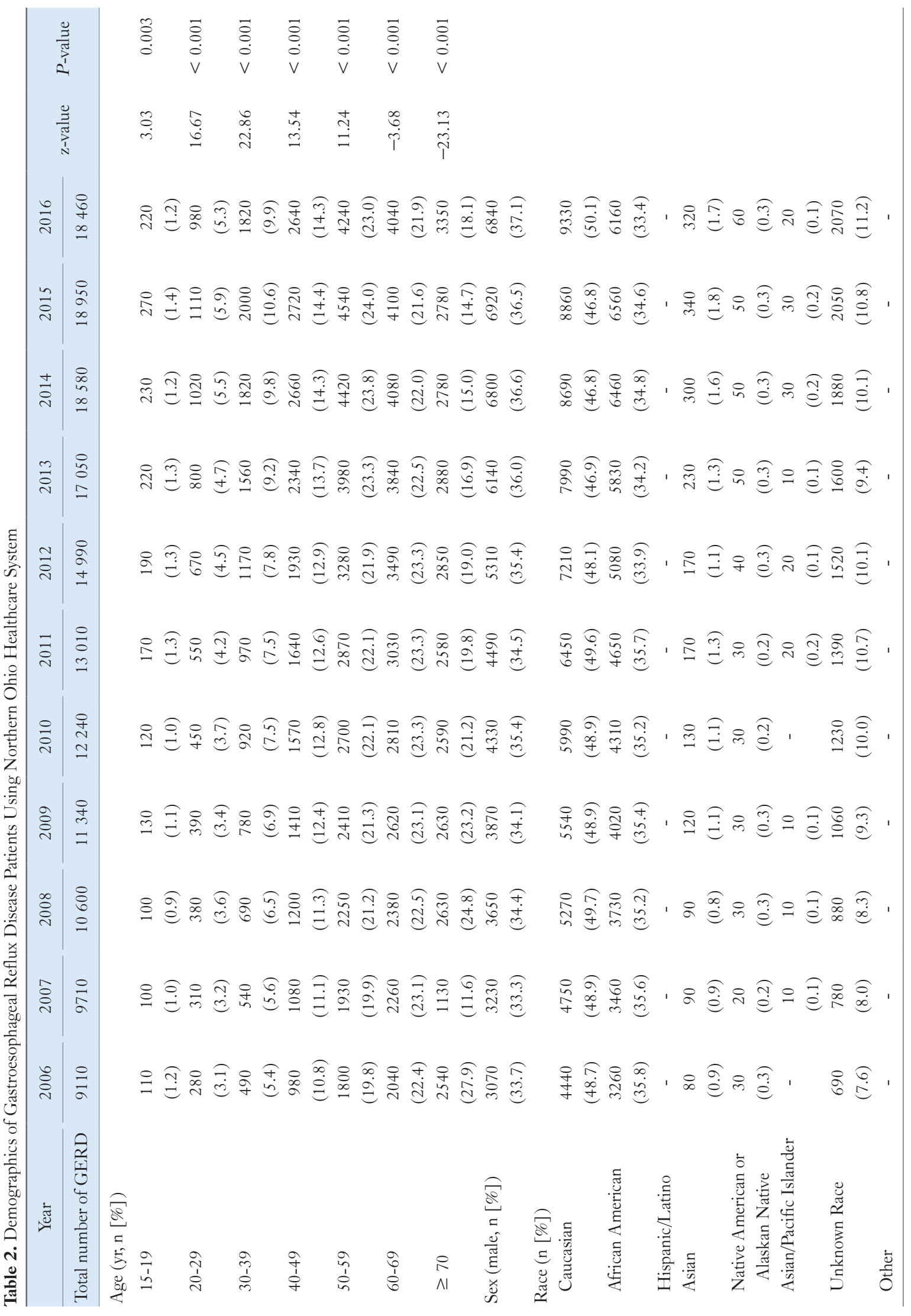




\begin{tabular}{|c|c|c|c|}
\hline 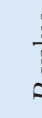 & & & 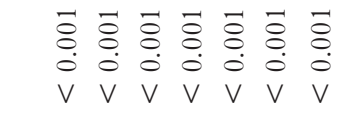 \\
\hline & & & 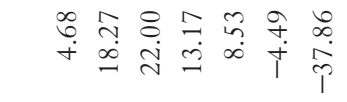 \\
\hline$\stackrel{\text { i }}{\pi}$ & \begin{tabular}{l}
8 \\
\multirow{1}{\infty}{} \\
$\stackrel{-}{\sim}$
\end{tabular} & 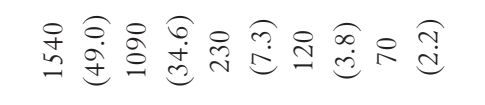 & 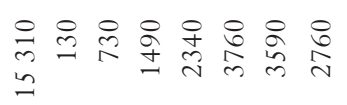 \\
\hline$\stackrel{n}{\vec{n}}$ & 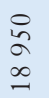 & 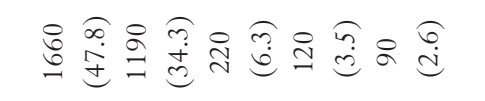 & 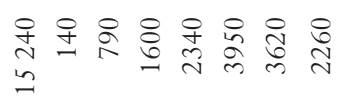 \\
\hline$\frac{\text { I }}{\vec{s}}$ & $\begin{array}{l}\infty \\
\infty \\
\infty \\
\infty \\
\sim\end{array}$ & 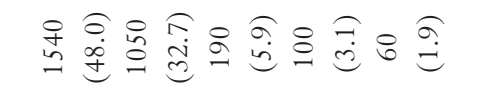 & 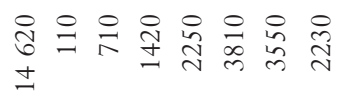 \\
\hline$\stackrel{\text { m }}{\stackrel{m}{2}}$ & 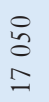 & 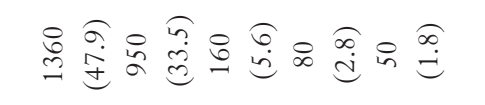 & 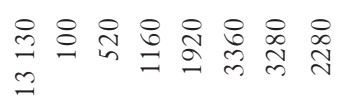 \\
\hline$\underset{\sim}{\sim}$ & $\begin{array}{l}\text { \& } \\
\pm \\
\pm\end{array}$ & 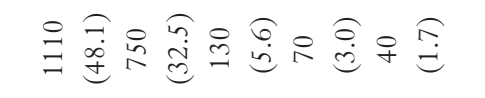 & 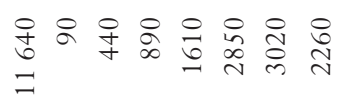 \\
\hline $\overrightarrow{\text { సे }}$ & $\begin{array}{l}\circ \\
0 \\
-2\end{array}$ & 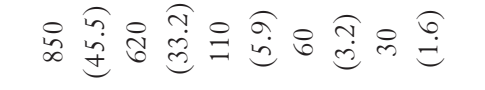 & 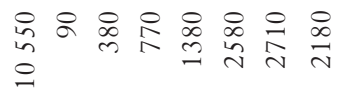 \\
\hline 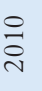 & $\underset{\substack{\text { I } \\
\text { I }}}{\simeq}$ & 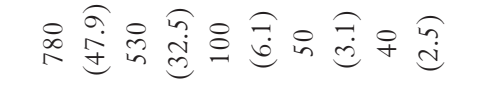 & 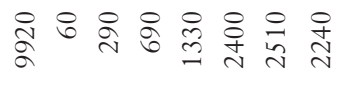 \\
\hline ठे̀े & $\begin{array}{l}\text { qे } \\
\text { m } \\
=\end{array}$ & 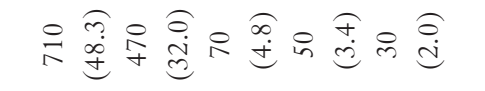 & $\begin{array}{l}0 \\
\vec{a} \\
\cdots\end{array}$ \\
\hline 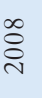 & $\begin{array}{l}8 \\
\stackrel{8}{0} \\
0\end{array}$ & 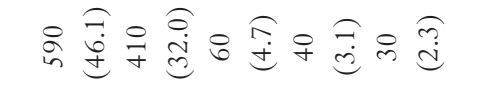 & 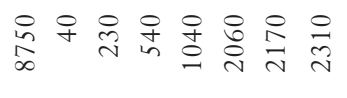 \\
\hline ڤ્े & $\stackrel{\circ}{\stackrel{2}{ล}}$ & 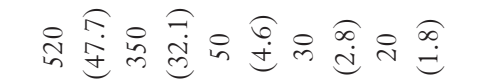 & 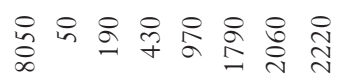 \\
\hline ๕ั̀ & $\begin{array}{l}\stackrel{Ð}{\sigma} \\
\sigma\end{array}$ & 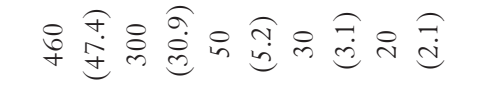 & 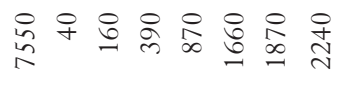 \\
\hline 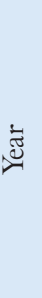 & 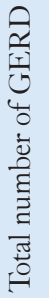 & 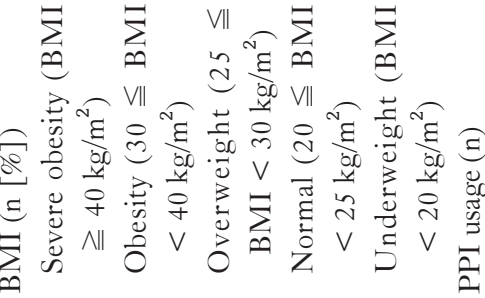 & 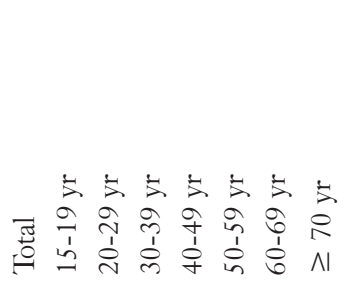 \\
\hline
\end{tabular}


were aged $\geq 70$ years in both the universal and Healthcare system datasets. In the system dataset, it was also found that the proportion of patients diagnosed with GERD has been decreasing in those who are aged $\geq 60$ years.

GERD has been considered to be a disease of middle aged and older subjects. One study demonstrated that the prevalence of gastroesophageal reflux symptoms was significantly higher in subjects aged $\geq 50$ years as compared with those who were aged $<50$ years (OR, 1.32; 95\% CI, 1.12-1.54; $P<0.001) .{ }^{10}$ In a populationbased cohort study from Norway, participants were evaluated for the degree of their GERD symptoms during the past 12 months. The study demonstrated that the incidence of GERD-related symptoms increased with age. ${ }^{14}$ Despite the results of our study, the age groups 60-70 years and $\geq 70$ years remained the largest as compared with all other age groups (as has been shown by the aforementioned studies).

In our study, however, the greatest rise in the proportion of patients with GERD diagnosis was seen in young adults aged 30-39 years in both datasets during the last decade. These results clearly suggest that younger subjects are more exposed today to risk factors for GERD development as compared with 10 years ago. In addition, it appears that risk factors for GERD continue to affect a growing number of the adult population—but specifically younger subjects, with resulting in early development of GERD. In a population-based study, more than $30.0 \%$ of heartburn sufferers reported reduced work productivity, with younger age being associated with these findings. ${ }^{15}$ Another retrospective study demonstrated that reflux esophagitis is present in $28.7 \%$ of all patients between the ages of 1 month and 20 years who underwent an upper endoscopy, which is a dramatic increase over the past 14 years. ${ }^{16}$ Interestingly, several studies have demonstrated that erosive reflux disease symptoms, such as heartburn and regurgitation, decrease in severity with aging. $6,13,17,18$

An important finding of our study was the general characteristic of GERD patients who were primarily obese or severely obese, older women, and Caucasian. These findings are consistent with several epidemiological studies, suggesting that GERD is more common in overweight/obese subjects, Caucasians, and older women. ${ }^{19}$ While erosive esophagitis and Barrett's esophagus (the more severe presentations of GERD) are more common in overweight/ obese subjects, Caucasians, and older males, most GERD patients have nonerosive reflux disease (NERD), which is primarily a female disease explaining the results of our study. ${ }^{20}$ NERD accounts for $60-70 \%$ of the GERD patients. ${ }^{21}$

In our study, the proportion of GERD patients using PPIs fell significantly in those who were over 70 years old in the universal dataset. In contrast, the proportion of GERD patients using PPIs has significantly increased in the other age groups, with the greatest increase being in the 30-39 years old group. Both trends closely follow the incidence trends of GERD in the same age groups. While PPI consumption remains high, with Americans spending more than 10 billion dollars per year for the different PPIs, concerns have been raised about the possible development of side effects. Based on our study results, more GERD patients are starting on chronic PPI treatment at a younger age, which may potentially increase the likelihood of long-term adverse events such as chronic kidney disease, osteoporosis, gastrointestinal infection, pneumonia, and others. ${ }^{22}$

Our study has several limitations that need to be discussed. The results are based on the Explorys dataset, which originates from 26 major Healthcare systems and 360 hospitals overall. This vast dataset is de-identified and thus may not reveal regional or individual hospital trends. Contributions to the dataset originate from many hospitals around the country, but this may not truly represent the diverse Healthcare system in the United States. Furthermore, the diagnosis of GERD was based on the term "Diagnosis: gastroesophageal reflux disease" as the cohort criterion without clarifying the severity of symptoms. Thus, we could not determine the relationship between severity of GERD and age. Consequently, it is assumed that GERD was diagnosed based on endoscopic findings or patient symptoms. In addition, Explorys is susceptible to limited documentation by physicians, which can lead to error in data collection and confound analysis.

In conclusion, GERD remains predominantly a disease of the middle aged and elderly with a higher proportion among Caucasians and females. However, there has been a significant increase in the proportion of patients with GERD in the younger age groups. Support for our findings comes from the proportion of patients with GERD who were using PPIs in our patient population. Our study suggests that physicians should be more aware that the proportion of young adults with GERD has been increasing continuously. It is also possible —although not evaluated in our study — that the proportion of young adults among patients with erosive esophagitis or Barrett's esophagus has been increasing as well. The ramifications of the aforementioned trend remain to be elucidated.

\section{Financial support: None.}

Conflicts of interest: None.

Author contributions: Ronnie Fass: study design, data analysis, 
and drafting and finalizing manuscript; Takahisa Yamasaki, Colin Hemond, and Mohamed Eisa: data collection, data analysis, and drafting and finalizing manuscript; and Stephen Ganocy: data analysis, drafting and finalizing manuscript, and statistical analysis.

\section{References}

1. Tack J, Becher A, Mulligan C, Johnson DA. Systematic review: the burden of disruptive gastro-oesophageal reflux disease on health-related quality of life. Aliment Pharmacol Ther 2012;35:1257-1266.

2. El-Serag HB, Sweet S, Winchester CC, Dent J. Update on the epidemiology of gastro-oesophageal reflux disease: a systematic review. Gut 2014;63:871-880.

3. Mahadeva S, Raman MC, Ford AC, et al. Gastro-oesophageal reflux is more prevalent in Western dyspeptics: a prospective comparison of British and South-East Asian patients with dyspepsia. Aliment Pharmacol Ther 2005;21:1483-1490.

4. Jarosz M, Taraszewska A. Risk factors for gastroesophageal reflux disease: the role of diet. Prz Gastroenterol 2014;9:297-301.

5. Saberi-Firoozi M, Khademolhosseini F, Yousefi M, Mehrabani D, Zare N, Heydari ST. Risk factors of gastroesophageal reflux disease in Shiraz, southern Iran. World J Gastroenterol 2007;13:5486-5491.

6. Lee J, Anggiansah A, Anggiansah R, Young A, Wong T, Fox M. Effects of age on the gastroesophageal junction, esophageal motility, and reflux disease. Clin Gastroenterol Hepatol 2007;5:1392-1398.

7. Achem AC, Achem SR, Stark ME, DeVault KR. Failure of esophageal peristalsis in older patients: association with esophageal acid exposure. Am J Gastroenterol 2003;98:35-39.

8. Ter RB, Johnston BT, Castell DO. Influence of age and gender on gastroesophageal reflux in symptomatic patients. Dis Esophagus 1998;11:106-108.

9. Becher A, Dent J. Systematic review: ageing and gastro-oesophageal reflux disease symptoms, oesophageal function and reflux oesophagitis. Aliment Pharmacol Ther 2011;33:442-454.

10. Eusebi LH, Ratnakumaran R, Yuan Y, Solaymani-Dodaran M, Bazzoli F, Ford AC. Global prevalence of, and risk factors for, gastro-oesophageal reflux symptoms: a meta-analysis. Gut 2018;67:430-440.

11. Thukkani N, Sonnenberg A. The influence of environmental risk factors in hospitalization for gastro-oesophageal reflux disease-related diagnoses in the United States. Aliment Pharmacol Ther 2010;31:852-861.

12. Sonnenberg A, Massey BT, Jacobsen SJ. Hospital discharges resulting from esophagitis among Medicare beneficiaries. Dig Dis Sci 1994;39:183-188.

13. Johnson DA, Fennerty MB. Heartburn severity underestimates erosive esophagitis severity in elderly patients with gastroesophageal reflux disease. Gastroenterology 2004;126:660-664.

14. Ness-Jensen E, Lindam A, Lagergren J, Hveem K. Changes in prevalence, incidence and spontaneous loss of gastro-oesophageal reflux symptoms: a prospective population-based cohort study, the HUNT study. Gut 2012;61:1390-1397.

15. Dean BB, Crawley JA, Schmitt CM, Wong J, Ofman JJ. The burden of illness of gastro-oesophageal reflux disease: impact on work productivity. Aliment Pharmacol Ther 2003;17:1309-1317.

16. Matsuura B, Nunoi H, Miyake T, Hiasa Y, Onji M. Obesity and gastrointestinal liver disorders in Japan. J Gastroenterol Hepatol 2013;28(suppl 4):48-53.

17. Pilotto A, Franceschi M, Leandro G, et al. Clinical features of reflux esophagitis in older people: a study of 840 consecutive patients. J Am Geriatr Soc 2006;54:1537-1542.

18. Maekawa T, Kinoshita Y, Okada A, et al. Relationship between severity and symptoms of reflux oesophagitis in elderly patients in Japan. J Gastroenterol Hepatol 1998;13:927-930.

19. Craven MR, Kia L, O’Dwyer LC, Stern E, Taft TH, Keefer L. Systematic review: methodological flaws in racial/ethnic reporting for gastroesophageal reflux disease. Dis Esophagus Published Online First: 1 Mar 2018. doi: 10.1093/dote/dox154.

20. Hershcovici T, Fass R. Nonerosive reflux disease (NERD) - an update. J Neurogastroenterol Motil 2010;16:8-21.

21. Fass R, Fennerty MB, Vakil N. Nonerosive reflux disease--current concepts and dilemmas. Am J Gastroenterol 2001;96:303-314.

22. Vaezi MF, Yang YX, Howden CW. Complications of proton pump inhibitor therapy. Gastroenterology 2017;153:35-48. 